\title{
Direct Calculation of Solid-Liquid Interfacial Free Energy for Molecular Systems: TIP4P Ice-Water Interface
}

\author{
Richard Handel and Ruslan L. Davidchack \\ Department of Mathematics, University of Leicester, Leicester, LE1 7RH, UK \\ Jamshed Anwar and Andrey Brukhno \\ Computational Laboratory, Institute of Pharmaceutical Innovation, \\ University of Bradford, West Yorkshire BD7 1DP, UK
}

(Dated: November 29, 2007)

\begin{abstract}
By extending the cleaving method of Broughton and Gilmer [J. Chem. Phys. 84, 5759 (1986)] to molecular systems we perform direct calculations of the ice $\mathrm{I}_{h}$-water interfacial free energy at ambient pressure for the TIP4P model. The values for the basal, prism, and $\{11 \overline{2} 0\}$ faces of ice $\mathrm{I}_{h}$ are determined to be $23.3 \pm 0.8 \mathrm{~mJ} \mathrm{~m}^{-2}, 23.6 \pm 1.0 \mathrm{~mJ} \mathrm{~m}^{-2}$, and $24.7 \pm 0.8 \mathrm{~mJ} \mathrm{~m}^{-2}$, respectively. The closeness of these values implies a minimal role of thermodynamic factors in determining the anisotropic behaviour observed during ice nucleation. These results are about $20 \%$ lower than the best experimentally-based estimates. However, we observe a larger discrepancy in the Turnbull coefficient, which is about $50 \%$ higher than for real water, indicating a possible limitation of the TIP4P model in describing the freezing transition.
\end{abstract}

PACS numbers: 68.08.-p,64.70.Dv,05.70.Np,87.15.Aa

Introduction.-The excess free energy of a solid-liquid interface, $\gamma_{\mathrm{sl}}$, is the reversible work required to form a unit area of interface between the two phases. A knowledge of this quantity is essential for understanding solidliquid phase transitions, including the processes of nucleation and crystal growth [1]. In a supercooled liquid, $\gamma_{\mathrm{sl}}$ counteracts the effects of the free energy difference between solid and liquid phases, and hence determines the size of the critical nucleus. The anisotropy of $\gamma_{\mathrm{sl}}$, i.e., its dependence on the orientation of the interface with respect to the crystal lattice, influences the shape of the crystal and the morphology of dendritic growth [2]. Clearly, being able to accurately determine $\gamma_{\mathrm{sl}}$ and its anisotropy is of considerable fundamental and technological interest.

Recently, there has been much progress in the development of methodology for determining $\gamma_{\mathrm{sl}}$ and its anisotropy by means of molecular simulation. Two distinct but complementary methods have been developed: the cleaving method, introduced by Broughton and Gilmer [3] and subsequently enhanced by Davidchack and Laird [4-6], and the capillary fluctuation method (CFM) proposed by Hoyt, Asta, and Karma [7]. The cleaving method uses external potentials to reversibly transform two separate solid and liquid systems prepared at coexistence conditions into a single system with the two phases juxtaposed to create an interface. The value of $\gamma_{\mathrm{sl}}$ is obtained directly by measuring the work performed by the external potentials during the transformation process. The main technical difficulty with this method is that it involves several stages, each of which requires precise control to ensure the transformation proceeds reversibly. The implementation of CFM is more straightforward, since it only requires the simulation of an equilibrated interfacial system. However, in this case the value of $\gamma_{\mathrm{sl}}$ is obtained indirectly, relying on the validity of the relationship between the magnitude of the capillary fluctuations and the stiffness of the interface, which, in turn, is related to $\gamma_{\mathrm{sl}}$ through a functional dependence with a carefully chosen set of anisotropy parameters. The cleaving method is more precise in determining specific values of $\gamma_{\mathrm{sl}}$, while the CFM is more sensitive for ascertaining the anisotropy.

It is notable that all systems studied to date have been atomic, with the exception of succinonitrile (SCN) [8] and a hard-dumbbell system [9]. However, both of these systems are amenable to current methods, since SCN freezes into an orientationally disordered bcc crystal and hence behaves essentially as atomic, while the cleaving walls approach, developed originally for the hard-sphere system [4], is directly applicable to the hard-dumbbell system.

We report here a significant extension of the cleaving method that enables the direct calculation of the solidliquid interfacial free energy for molecular systems. The main challenge is to incorporate the orientational degrees of freedom into the transformation path, so that, in the process of forming the interface, the liquid molecules occupy crystal sites with appropriate orientational order. We solve this problem by designing a cleaving potential that influences both position and orientation of the molecules. The proposed potential is generic and can be applied to all types of rigid molecules, as well as being adaptable to flexible molecular systems. We use the extended cleaving method to determine the free energy of the interface between ice $\mathrm{I}_{h}$ and water at ambient pressure for the TIP4P model of water. Even though the ice $\mathrm{I}_{h}$ structure consists of orientationally disordered hydrogen bonds, the orientation of molecules during the cleaving process needs to be controlled in order for the crystal structure to obey the Bernall-Fowler ice rules [10].

The cleaving method. - The method consists of four 
basic steps: Step 1: Cleave the solid system with a suitably chosen external potential (the cleaving potential) along a plane (the cleaving plane) between two crystal layers of a given orientation; Step 2: Cleave the liquid system with a matching potential, which induces partial ordering of the liquid near the cleaving plane, and creates a barrier through which the liquid particles cannot cross; Step 3: Merge the two systems by gradually rearranging the boundary conditions to allow interaction between the solid and the liquid across the cleaving planes while maintaining the cleaving potential; Step 4: Remove the cleaving potential from the combined system.

Provided the initial isolated solid and liquid systems have been equilibrated at the solid-liquid coexistence conditions, the only result of the above transformation process is the creation of the solid-liquid interface. Therefore, the total work performed on the system constitutes the product of the interfacial free energy $\gamma_{\mathrm{sl}}$ and the area of the created interface.

The reversible work in each step is determined using a standard coupling parameter approach [11] wherein the total potential energy of the system depends on the coupling parameter $\lambda$, i.e. $U=U(\lambda)$, in such a way that changing the parameter value (typically from $\lambda=0$ to $\lambda=1$ ) transforms the system from its initial to its final state. In the present study we have opted to compute the reversible work in each of the four steps using the nonequilibrium work approach in the spirit of the Bennett acceptance ratio (BAR) method [12-16]. Within this approach, the coupling parameter $\lambda$ is changed continuously from its initial to its final value over the duration of the simulation run (or, if one were employing the Monte Carlo method, over a number of sweeps) [17]. Compared to the more traditional thermodynamic integration approach, the nonequilibrium BAR method appears to be more efficient and, even more significantly, provides a highly reliable estimate of the error on the determined free energy [15].

The cleaving potential.-The cleaving potential is introduced to prevent the particles from crossing the cleaving plane (otherwise the process of rearranging the boundary conditions in Step 3 is not well defined). A suitable choice of cleaving potential is crucial to the success of the method, since accurate results can only be obtained if the transformation path is near-reversible on the time scale of the simulation. Both requirements can be satisfied if the cleaving potential is designed to promote the formation of crystal layers at the cleaving plane in Step 2. In the previous implementations of the cleaving method, the cleaving potential has been purely repulsive, tailored to mimic the interaction of liquid particles with layers of crystal particles at the interface. Such a repulsive cleaving potential cannot be directly applied to systems where attractive forces (e.g. electrostatic interactions) play an essential role in determining the crystal structure. The task of designing the cleaving potential for molecular systems is further complicated by the need to induce specific orientations of the molecules within the crystal structure.

We construct the cleaving potential from a set of potential wells located at the ideal crystal positions near the cleaving plane. These wells attract the molecules in the liquid to the lattice sites and orientate them in a prescribed way to promote the formation of crystal layers. For a rigid molecule with center-of-mass coordinates $\mathbf{r}$ and orientational coordinates $\mathbf{q}$ (e.g. rotation matrix, Euler angles, or quaternions), the proposed cleaving potential has the following generic form:

$$
\Phi(\mathbf{r}, \mathbf{q})=\sum_{j} \phi\left(\left|\mathbf{r}-\mathbf{R}_{j}\right|\right) \theta\left(\mathbf{q}, \mathbf{Q}_{j}\right)
$$

where $\mathbf{R}_{j}$ is the position of the potential well $j$ and $\mathbf{Q}_{j}$ is the desired orientation of a molecule within the well. Typically, $\phi(r)$ can be any potential well function with a minimum (negative) value $-d_{\mathrm{w}}$ (the "well depth") at $r=0$ and a finite range $r_{\mathrm{w}}$, i.e., $\phi\left(r \geq r_{\mathrm{w}}\right)=0$. In this work we employed a simple polynomial function: $\phi(r)=$ $d_{\mathrm{w}}\left[\left(r / r_{\mathrm{w}}\right)^{2}-1\right]^{3}$ for $r<r_{\mathrm{w}}$.

To induce the desired orientation, $\theta(\mathbf{q}, \mathbf{Q})$ should be a smooth function which is positive when a molecule is aligned with the desired orientation (i.e., $\mathbf{q}$ is close to Q) and negative when the molecule is misaligned. The overall result is a potential which attracts molecules with good alignment, and repels those with bad alignment, and furthermore exerts a torque on the molecules in the direction of perfect alignment.

Application to TIP 4 P water.-We have applied the cleaving method with the proposed molecular cleaving potential to the TIP4P model of water [18] and computed ice $\mathrm{I}_{h}$-water interfacial free energy for the $\{0001\}$ (basal), $\{1 \overline{1} 00\}$ (prism), and $\{11 \overline{2} 0\}$ interfaces. Both the Lennard-Jones and electrostatic interactions were truncated (discontinuously) at $10 \AA$. We employed molecular dynamics simulation in the NVT ensemble using a quaternion-based algorithm NO_SQUISH [19] with 2 fs time step. The temperature was controlled using the Nosé-Hoover method, with separate control of translational and rotational motions. In order to determine the coexistence conditions for this water model, trial equilibration runs of the ice $\mathrm{I}_{h}$-water interface systems were performed at different temperatures [20]. We found the run at $219 \mathrm{~K}$ to be closest to coexistence, exhibiting no overall freezing or melting over a $4 \mathrm{~ns}$ simulation run. This temperature is lower than the $232 \mathrm{~K}$ melting temperature of TIP4P water reported in [21], and the $229 \mathrm{~K}$ reported in [22] and [23]. This discrepancy is the result of differences in the handling of electrostatic interactions: [21] used Ewald sums, while [22] and [23] used a cutoff of $17 \AA$, compared to our $10 \AA$. Further details of the methodology employed by us to ascertain the melting temperature for our system can be found in [17]. The coexistence densities of $938.0 \mathrm{~kg} / \mathrm{m}^{3}$ for ice $\mathrm{I}_{h}$ and $1010.8 \mathrm{~kg} / \mathrm{m}^{3}$ for water were found to yield the approximate ambient pressure of 1 bar at $219 \mathrm{~K}$. 
Separate equilibrium ice and water systems were prepared at the coexistence conditions. The protondisordered structure of ice $\mathrm{I}_{h}$ was generated using the algorithm of Buch et al. [24]. The ice system contained approx. 2100 molecules in a simulation cell of dimensions $L_{x} \approx 44 \AA, L_{y} \approx 39 \AA, L_{z} \approx 45 \AA$. The water system with exactly the same $L_{x}$ and $L_{y}$ dimensions (the cleaving plane is chosen normal to the $z$-axis) had approx. 2400 molecules and $L_{z} \approx 42 \AA$.

The orientational part of the cleaving potential in Eq. (1) took the form:

$$
\theta(\mathbf{q}, \mathbf{Q})=\mathbf{n}(\mathbf{q}) \cdot \mathbf{n}(\mathbf{Q})
$$

where, $\mathbf{n}$ is a unit vector directed from the oxygen to the mid-point of the hydrogens. Note that the torque induced by $\theta$ only influences two of the three rotational degrees of freedom; our studies confirm that this is sufficient inducement for the molecule to find its correct orientation.

In the cleaving step for ice (Step 1), two layers of potential wells are introduced at the ideal crystal positions on either side of the cleaving plane, with orientations $\mathbf{Q}$ aligned with the orientations of the crystal molecules at the wells.

The potential for cleaving the liquid (Step 2) is identical to the potential for cleaving the solid. As noted in previous studies $[5,6]$, the structural ordering of the liquid induced by the cleaving potential is the principal source of irreversibility in the cleaving method. For the TIP4P water system, the hysteresis was found to be very persistent and could not be removed by slowing down the switching process, but could be reduced significantly by cleaving at a higher temperature. So cleaving the liquid becomes a 3 -stage process: (1) heat the system, (2) cleave the heated system, (3) re-cool the system. We found it sufficient to heat the system to about $310 \mathrm{~K}$. Instead of adjusting the simulation temperature, we adopted the equivalent approach of scaling down the potential [25]. The scaling work is then calculated in a similar way to the cleaving work. The amount of hysteresis is reliably quantified by the nonequilibrium BAR measurement approach, since the presence of hysteresis in the switching process gives rise to a substantial increase in the BAR error estimator. See [17] for details. An illustration of the cleaved liquid system is shown in Figure 1, where the cleaving potential has induced the formation of a layer of ice $\mathrm{I}_{h}$ crystal for the basal orientation of the interface.

To merge the two systems (Step 3), the combined potential $U$ is controlled by a coupling parameter $\lambda$ as follows: $U(\lambda)=(1-\lambda)\left(U_{\mathrm{i}}+U_{\mathrm{w}}\right)+\lambda U_{\mathrm{iw}}$, where $U_{\mathrm{i}}$ and $U_{\mathrm{w}}$ are the molecular interaction potentials of separate ice and water systems and $U_{\mathrm{iw}}$ is the potential of the combined system. Finally, the cleaving potential is removed from the combined ice-water system (Step 4).

The interfacial free energy is given by the sum of the work performed in each of Steps 1-4 divided by the area of the created interface, $A=2 L_{x} L_{y}$. The computed values of the ice $\mathrm{I}_{h}$-water interfacial free energy for

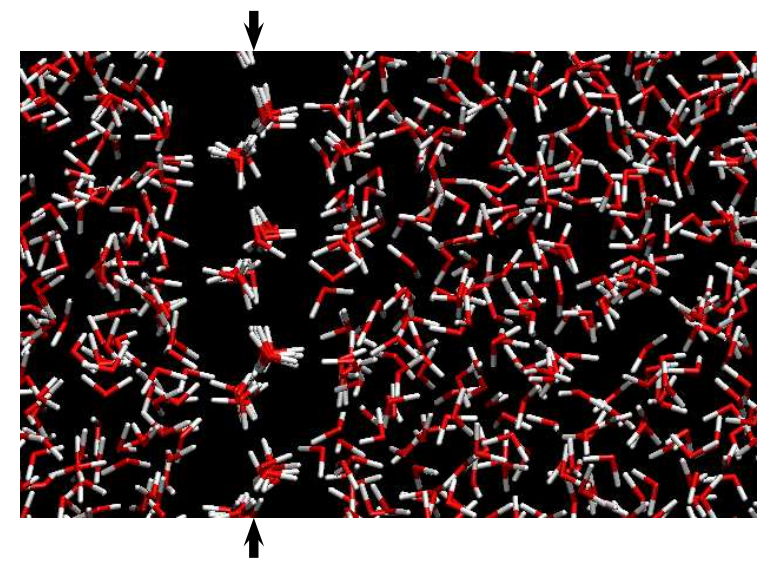

FIG. 1: (Color online) TIP4P water system at the end of Step 2 (basal interface). Arrows indicate the location of the cleaving plane.

TIP4P were $23.3 \pm 0.8 \mathrm{~mJ} \mathrm{~m}^{-2}$ for the basal face, $23.6 \pm$ $1.0 \mathrm{~mJ} \mathrm{~m}^{-2}$ for the prism face, and $24.7 \pm 0.8 \mathrm{~mJ} \mathrm{~m}^{-2}$ for the $\{11 \overline{2} 0\}$ face of ice $\mathrm{I}_{h}$.

Discussion.-Because of its importance, there have been numerous attempts to determine the interfacial free energy of the ice-water interface by experiment using a variety of methods (see Ref. [26] for a review of experimental results). Even though the scatter in these results exceeds $50 \%$, the more recent estimates $[26,27]$ tend to converge to the value $29.1 \pm 0.8 \mathrm{~mJ} \mathrm{~m}^{-2}$ obtained by Hardy using the shape of the grain boundary groove method [28]. So far, the computer simulation results for $\gamma_{\mathrm{sl}}$ for ice-water interface have been obtained only by indirect methods: Haymet, Bryk, and Smith [29] determined the interfacial tension along the basal face for the SPC/E model to be $39 \pm 4 \mathrm{~mJ} \mathrm{~m}^{-2}$ and argued that the difference between the tension and the free energy of the ice-water interface should be small [38]. In a recent study, Wang, Tang and Zeng [30] used the superheatingundercooling hysteresis method [27] to determine $\gamma_{\mathrm{sl}}$ for TIP4P-Ew and TIP5P-Ew models and obtained values of $37 \pm 3 \mathrm{~mJ} \mathrm{~m}^{-2}$ and $42 \pm 4 \mathrm{~mJ} \mathrm{~m}^{-2}$, respectively.

Calculation of the Turnbull coefficient [31], $C_{T}$, reveals that the lower than experimental $\gamma_{s 1}$ values we obtained could be attributed to the low enthalpy of fusion, $\Delta H_{\text {fus }}$, of the water model employed in our study. The Turnbull coefficient, defined by $\gamma_{\mathrm{sl}} \rho_{\mathrm{s}}^{-2 / 3}=C_{T} \Delta H_{\text {fus }}$, where $\rho_{\mathrm{s}}$ is the number density of the solid phase, commonly takes a value of about 0.45 for metals, and 0.32 for many non-metallic materials. Since for our water model $\Delta H_{\text {fus }}=3.18 \mathrm{~kJ} \mathrm{~mol}^{-1}$, whilst for real water $\Delta H_{\text {fus }}=$ $6.02 \mathrm{~kJ} \mathrm{~mol}^{-1}$, our Turnbull coefficient, $C_{T}=0.45$, is substantially higher than that of real water, $C_{T}=0.30$. The low value of $\Delta H_{\text {fus }}$ is partially the result of truncation of the electrostatic interactions in our model, since TIP4P with full electrostatic interactions has an enthalpy of fusion $\Delta H_{\text {fus }}=4.39 \mathrm{~kJ} \mathrm{~mol}^{-1}$ at its coexistence temperature of $232 \mathrm{~K}$ [21]. Since $C_{T}$ is typically insensitive to the details of the interaction potential, we expect that for TIP4P with full electrostatics the ice-water interfacial free energy will be higher. The discrepancy for $C_{T}$ 
between TIP4P and real water might indicate a limitation of this model in describing freezing. It remains to be investigated whether inclusion of full electrostatic interactions will reduce this discrepancy.

The dependence of $\gamma_{\mathrm{sl}}$ on the crystal face at the interface remains poorly characterised. Experimentally, the ratios could be estimated from the equilibrium shape of an ice $\mathrm{I}_{h}$ crystal using the well known Wulff construction [32] but, to our knowledge, the equilibrium shapes of isolated ice crystals have never been definitively observed [33]. The experimental observation of the oblate ellipsoidal shape of water inclusions in ice $\mathrm{I}_{h}[34]$ would imply a very large ratio $\gamma_{\text {prism }} / \gamma_{\text {basal }}=1.857$, but this could also be attributed to the strong influence of mechanical stresses on the shape of the inclusions [35]. According to a rough estimate based on the number of broken bonds at the crystal surface [36, 37] $\gamma_{\text {basal }}: \gamma_{\text {prism }}: \gamma_{\{11 \overline{2} 0\}} \approx 1: 1.06: 1.22$. Our results are consistent with this estimate in that the value for $\gamma_{\{11 \overline{2} 0\}}$ is higher than the values for the other two interfaces, which are marginally smaller but very similar. The similarity between the calculated interfacial free energies for different crystallographic planes implies a minimal role of thermodynamic factors in determining the anisotropic behaviour observed during ice nucleation.

Conclusion.-We have extended the cleaving method to molecular systems and determined the TIP4P ice $\mathrm{I}_{h^{-}}$ water interfacial free energy for the basal, prism, and $\{11 \overline{2} 0\}$ orientations to be $23.3 \pm 0.8 \mathrm{~mJ} \mathrm{~m}^{-2}, 23.6 \pm$ $1.0 \mathrm{~mJ} \mathrm{~m}^{-2}$, and $24.7 \pm 0.8 \mathrm{~mJ} \mathrm{~m}^{-2}$, respectively. In our future work, we will investigate the influence of electrostatic potential truncation on $\gamma_{\mathrm{sl}}$ and $C_{T}$ by including full electrostatic interactions (e.g., via Ewald sums) in our calculations. We will also compute $\gamma_{\mathrm{sl}}$ for other models of water and other ice phases, as well as extend the cleaving method to flexible molecular model potentials.

We would like to thank Brian Laird, Taras Bryk, and László Gránásy for helpful comments and discussion. This work was supported by the EPSRC Grants GR/T27105/01 and GR/T27112/01. The computations were performed on the University of Leicester Mathematical Modelling Centre's cluster, which was purchased through the EPSRC strategic equipment initiative.

[1] W. A. Tiller, The Science of Crystallization: Microscopic Interfacial Phenomena (Cambridge University Press, New York, 1991).

[2] J. J. Hoyt, M. Asta, and A. Karma, Mat. Sci. Eng. R 41, 121 (2003).

[3] J. Q. Broughton and G. H. Gilmer, J. Chem. Phys. 84, 5759 (1986).

[4] R. L. Davidchack and B. B. Laird, Phys. Rev. Lett. 85, 4751 (2000).

[5] R. L. Davidchack and B. B. Laird, J. Chem. Phys. 118, 7651 (2003).
[6] R. L. Davidchack and B. B. Laird, Phys. Rev. Lett. 94, $086102(2005)$.

[7] J. J. Hoyt, M. Asta, and A. Karma, Phys. Rev. Lett. 86, 5530 (2001).

[8] X. Feng and B. Laird, J. Chem. Phys. 124, 044707 (2006).

[9] Y. Mu and X. Song, Phys. Rev. E 74, 031611 (2006).

[10] J. D. Bernal and R. H. Fowler, J. Chem. Phys. 1, 515 (1933).

[11] D. Frenkel and B. Smit, Unverstanding Molecular Simulation: From Algorithms to Applications (Academic Press, San Diego, 2002), 2nd ed.

[12] C. H. Bennett, J. Comput. Phys. 22, 245 (1976).

[13] G. E. Crooks, Phys. Rev. E 61, 2361 (2000).

[14] M. R. Shirts, V. S. Pande, E. Bair, and G. Hooker, Phys. Rev. Lett. 91, 140601 (2003).

[15] M. R. Shirts and V. S. Pande, J. Chem. Phys. 122, 144107 (2005).

[16] Y. Mu and X. Song, J. Chem. Phys. 124, 034712 (2006).

[17] See EPAPS Document No. [number inserted by publisher] for more details. For more information on EPAPS, see http://www.aip.org/pubservs/epaps.html.

[18] W. L. Jorgensen, J. Chandrasekhar, J. D. Madura, R. W. Impey, and M. L. Klein, J. Chem. Phys. 79, 926 (1983).

[19] T. F. Miller III, M. Eleftheriou, P. Pattnaik, A. Ndirango, D. Newns, and G. J. Martyna, J. Chem. Phys. 116, 8649 (2002).

[20] R. G. Fernández, J. L. F. Abascal, and C. Vega, J. Chem. Phys. 124, 144506 (2006).

[21] C. Vega, E. Sanz, and J. L. F. Abascal, J. Chem. Phys. 122, 114507 (2005).

[22] J. Wang, S. Yoo, J. Bai, J. R. Morris, and X. C. Zeng, J. Chem. Phys. 123, 036101 (2005).

[23] Y. Koyama, H. Tanaka, G. Gao, and X. C. Zeng, J. Chem. Phys. 121, 7926 (2004).

[24] V. Buch, P. Sandler, and J. Sadlej, J. Phys. Chem. B 102, 8641 (1998).

[25] M. de Koning, A. Antonelli, and S. Yip, Phys. Rev. Lett. 83, 3973 (1999).

[26] L. Gránásy, T. Pusztai, and P. F. James, J. Chem. Phys. 117, 6157 (2002).

[27] S.-N. Luo, A. Strachan, and D. C. Swift, Modelling Simul. Mater. Sci. Eng. 13, 321 (2005).

[28] S. C. Hardy, Philos. Mag. 35, 471 (1977).

[29] A. Haymet, T. Bryk, and E. Smith, in Ionic soft matter: Modern trends in theory and applications, edited by D. Henderson, M. Holovko, and A. Trokhymchuk (Kluwer, The Netherlands, 2005), pp. 333-359.

[30] J. Wang, Y. W. Tang, and X. C. Zeng, J. Chem. Theory Comput 3, 1494 (2007).

[31] D. Turnbull, J. Appl. Phys. 21, 1022 (1950).

[32] G. Wulff, Z. Kristallogr. Mineral. 34, 449 (1901).

[33] K. G. Libbrecht, Rep. Prog. Phys. 68, 855 (2005).

[34] K.-K. Koo, R. Ananth, and W. N. Gill, Phys. Rev. A 44, 3782 (1991).

[35] L. Gránásy, J. Mol. Struct. 485-486, 523 (1999).

[36] T. Kuroda and R. Lacmann, J. Crystal Growth 56, 189 (1982).

[37] T. Takahashi, J. Crystal Growth 59, 441 (1982).

[38] The equality between tension and free energy is exact for liquid-liquid and liquid-vapor interfaces, while for interfaces involving solids, the two quantities may differ due to the ability of solids to support stress. 